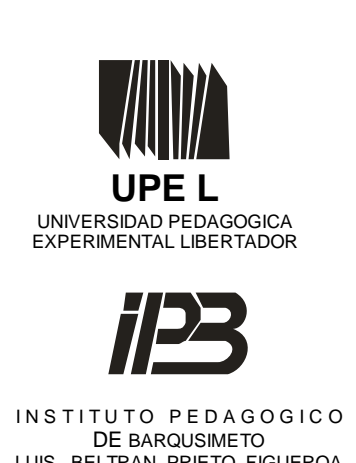

LUIS BELTRAN PRIETO FIGUEROA

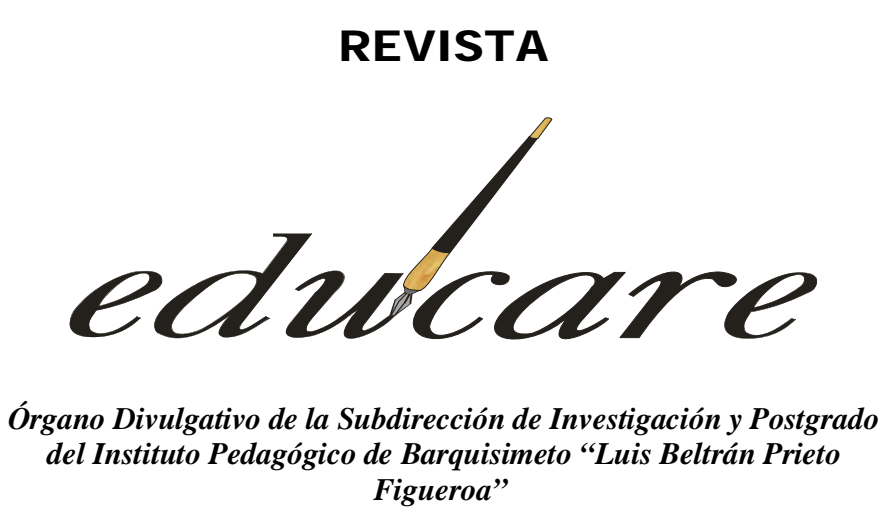

Figueroa”

BARQUISIMETO - EDO. LARA - VENEZUELA

NUEVA ETAPA

FORMATO ELECTRÒNICO

DEPOSITO LEGAL: ppi201002LA3674

Volumen $14 \mathrm{~N}^{\circ} 2$

ISSN: 2244-7296

Mayo-Agosto 2010

\title{
LA DIMENSIÓN AMBIENTAL EN EL COMPORTAMIENTO COTIDIANO DEL ESTUDIANTE DE LA TERCERA ETAPA DE EDUCACIÓN BÁSICA
}

ENVIRONMENTAL DIMENSION IN THE MIDDLE SCHOOL STUDENT'S DAILY BEHAVIOR

\author{
Orlando Silva \\ (Escuela Granja Hato Arriba) \\ Martín García \\ (UNY)
}




\title{
LA DIMENSION AMBIENTAL EN EL COMPORTAMIENTO COTIDIANO DEL ESTUDIANTE DE LA TERCERA ETAPA DE EDUCACION BASICA
}

\author{
ENVIRONMENTAL DIMENSION IN THE MIDDLE SCHOOL STUDENT'S DAILY \\ BEHAVIOR
}

TRABAJO DE INVESTIGACIÓN

Recibido:14-06-10

\author{
Orlando Silva* \\ Escuela Granja Hato Arriba \\ Martín García** \\ UNY
}

\begin{abstract}
RESUMEN
El artículo reporta una investigación que tuvo como propósito construir una aproximación interpretativa, desde la percepción de los actores de la comunidad educativa, sobre la dimensión ambiental en el comportamiento cotidiano del estudiante de la tercera etapa de educación básica, para lo cual se diseñó una investigación cualitativa, apoyada en el método fenomenológico. Se seleccionaron como informantes clave, de manera intencional, a diecinueve miembros de la comunidad educativa de la Escuela Granja Hato Arriba, ubicada en la comunidad del mismo nombre en el Municipio Morán del estado Lara. Entre los hallazgos se encuentran: el comportamiento incrongruente del estudiantado al depositar los residuos sólidos en sitios inadecuados con la correspondiente generación de conflictos entre el personal docente y de mantenimiento; la debilidad de compromiso por parte de los docentes para que la dimensión ambiental se practique como herramienta pedagógica que ayude a conservar el ambiente; y el bajo nivel de conciencia ambiental en la comunidad educativa. Se concluye en la necesidad de acitvar y fortalecer lazos de participación colectiva que ayuden a mejorar las relaciones interpersonales en pro de conservar su hábitat y elevar la conciencia ambientalista deseada.
\end{abstract}

Descriptores: comportamiento cotidiano, comportamiento ambiental, conservacion ambiental, educación ambiental, método fenomenológico
Aceptado: 30-07-10

\footnotetext{
* Técnico Superior Universitario en Tecnología Agrícola (IUTY, 1990), Profesor de Educación Agropecuaria (UPEL-IPB, 2003), Magister en Educación Ambiental (UNY, 2009). A la fecha se desempeña como Director de la Escuela Granja Hato Arriba, en el Municipio Morán del Estado Lara. Teléfono: 04245386133.

** Ingeniero Agrónomo (UCLA, 1974), Magíster en Ingeniería Hidráulica opción Suelos y Riego (ULA-CIDIAT, 1976), Doctor en Ciencias de la Educación (USM, 1999), Certificado de Estudios Posdoctorales en Gerencia (UNY, 2009). Actualmente forma parte del equipo de docentes investigadores del Instituto de Investigación y Postgrado de la Universidad Yacambú. Teléfono: 04163530866.
} 


\section{INTRODUCCIÓN}

El comportamiento humano en los distintos escenarios del mundo, derivado de las acciones antrópicas realizadas para la satisfacción de las necesidades biológicas, socioeconómicas y culturales, ha contribuido al deterioro ambiental en que se encuentra el globo terráqueo en la actualidad, dado el enfoque no sustentable de tales acciones que ha prevalecido hasta nuestros días. De esta manera, el mencionado comportamiento ha despertado un gran interés multidisciplinario, al tratar de conocer a través de la investigación las diferentes conductas del ser humano en su interacción con el entorno.

Aún cuando la problemática ambiental tiene una manifestación global, también tiene sus particularidades en cada país, como es el caso de Venezuela. En este sentido, el desequilibrio de los ecosistemas en el país se debe a factores extrínsecos e intrínsecos, en el primero de los casos, vinculados a los fenómenos naturales que han causado estragos en los espacios naturales tanto en sectores los urbanos como en los rurales; y en el segundo, por las actividades antrópicas donde el comportamiento humano individual o grupal se manifiesta en la mayoría de los casos mediante el desarrollo de acciones sin controles sociales, jurídicos, morales-conservacionistas y éticos-ambientalistas.

La situación ambiental antes referida, ha sido objeto de atención por distintos entes públicos y privados, mediante esfuerzos en materia de educación ambiental, materializados en diversas acciones, entre las cuales destacan por su importancia la inclusión de la dimensión ambiental en los currículos de los niveles de educación inicial, básica y diversificada, así como en programaciones diversas en el área educativa informal. Sin embargo, a pesar de tales esfuerzos, continúan observándose en el país serios problemas ambientales y comportamientos inadecuados de la ciudadanía en materia ambiental.

Tal es el caso de la comunidad educativa de la Escuela Granja Hato Arriba ubicada en el caserío Hato Arriba, distante 35 km de Humocaro Bajo, parroquia Morán en el municipio Morán del estado Lara, en la cual labora uno de los coautores como director y ha vivenciado comportamientos impropios en el estudiantado, no ajustados a los patrones de conducta esperados en un colectivo inmerso en un proceso de enseñanza y aprendizaje con la dimensión ambiental incorporada como eje transversal. 
Visto lo anterior, se realizó un trabajo de investigación cuyo propósito general fue construir un marco interpretativo, desde la percepción de los actores sociales de una comunidad educativa, sobre la dimensión ambiental como herramienta transformadora del comportamiento cotidiano en las y los estudiantes de la tercera etapa de educación básica de la Escuela Granja Hato Arriba, municipio Morán del estado Lara, enmarcado bajo el método de investigación fenomenológica. Los propósitos específicos fueron:

1. Indagar sobre la percepción de los actores educativos en cuanto a la dimensión ambiental del comportamiento cotidiano en el entorno de la comunidad educativa.

2. Interpretar la percepción de los actores educativos sobre la dimensión ambiental en el comportamiento cotidiano diferenciado en los distintos ámbitos de la comunidad educativa.

3. Construir las estructuras particulares y general sobre la dimensión ambiental en el comportamiento cotidiano de los actores educativos en la comunidad educativa, desde la percepción de tales actores.

\section{ORIENTACIONES TEÓRICAS}

Los estudios previos presentados en este capítulo y las orientaciones teóricas están vinculados con los hallazgos del trabajo, considerando el área temática en la cual se inserta la investigación realizada. Los contenidos expuestos permitieron efectuar las contrastaciones del caso con los hallazgos de otros autores, en función de la orientación fenomenológica de este estudio.

Entre los estudios previos relacionados con el tema de la investigación, se mencionan en el ámbito internacional autores como Moreno, Corraliza y Ruíz (2005), Fernández, Rodríguez y Carrazquer (2006), Pato y Tamayo (2006); en el ámbito nacional se citan a autores como Flen-Bers (2001), Escalona y Pérez (2006) y Gutiérrez (2007). En tal sentido, podemos indicar que las investigaciones anteriormente señaladas, tanto fuera y dentro del país, evidencian una clara inclinación de la preocupación marcada en la comunidad académica global y latinoamericana por la inserción de la educación ambiental en todos los niveles del sistema educativo como herramienta pedagógica para lograr transformar la conducta inapropiada de un individuo en atención a la preparación para 
solucionar los problemas ambientales en su ámbito local. Cabe mencionar la orientación metodológica cuantitativa de todas las investigaciones previamente señaladas, lo cual hace presumir sobre la necesidad de un abordaje cualitativo de estas temáticas, en aras de una mayor profundización interpretativa.

En cuanto a los referentes conceptuales, se presentan las respectivas construcciones, elaboradas a partir de la consulta a distintos autores (Uribe, Velasco y Escobar, 1991; Tréllez, 2000; Ferrer, 2001; Zaldívar, 2003; Baldi y García, 2005; Alea, 2005; Mora, 2005; Ley Orgánica del Ambiente, 2006; VITALIS, 2007; García, 2008; entre otros):

-Comportamiento Ambiental: Son actuaciones que el individuo realiza en pro de la preservación, conservación y mejoramiento de la calidad del ambiente para alcanzar una mejor calidad de vida.

-Comportamiento Grupal: Es la interacción de un colectivo que se desarrolla en torno a sus necesidades para producir hábitos positivos, valores, creencias que le permitirán el desarrollo de sus habilidades dentro de una organización social, donde se producirá el intercambio de conocimientos y experiencias para lograr el cambio de conducta según sus normas.

-Comportamiento Individual: Se refiere a la actuación del sujeto en relación a su hábitat, a su entorno natural donde se desarrolla con respecto a sus necesidades, donde debe combinar sus necesidades con los valores para aumentar su capacidad de adaptación. Asimismo, la conducta del individuo se relaciona con la necesidad de cubrir las expectativas de grupo y tener éxito en lo personal.

-Conducta Cotidiana: Conjunto de procedimientos y acciones que realizan los seres vivos como rutina diaria; esta conducta está asociada a factores intrínsecos y extrínsecos del entorno para adaptarse al medio donde se desarrolla el individuo.

-Conservación Ambiental: Proceso donde la interacción del ser humano con la naturaleza realiza cambios que son de dos tipos: positivos y negativos. En tal sentido, se trata de la protección y sostenibilidad del individuo para perpetuarse armonizando con los recursos naturales para mejorar la calidad de vida. Este proceso se considera multidisciplinario, con un carácter tanto individual como colectivo en la transformación y concienciación de los autores sociales. 
-Educación Ambiental: Es el instrumento que permite capacitar al individuo para adquirir los conocimientos ambientales con sus valores, además, de fomentar aptitudes y actitudes que son fundamentales para comprender y apreciar las interrelaciones entre el ser humano, su medio físico y su cultura.

-Psicología Ambiental: Es la ciencia que se encarga de estudiar el comportamiento del individuo hacia el ambiente, donde se pone de manifiesto las costumbres, actitudes, aptitudes, cultura y los valores ambientalistas del hombre en su interacción con el medio que lo rodea.

-Psicología Social: Esta se refiere al estudio del comportamiento del individuo como ser único y su relación con sus semejantes dentro del entorno. Asimismo estudia el comportamiento social en relación a su hábitat.

\section{ASPECTOS METODOLÓGICOS}

\section{El Contexto}

El caserío Hato Arriba se ubica dentro de un paisaje montañoso y de valles altos, situado en las estribaciones andinas del estado Lara, en municipio Morán del estado Lara en el kilómetro 35 de la vía que parte hacia esta localidad desde la ciudad de El Tocuyo. En cuanto a las potencialidades predominantes para el desarrollo económico, se destaca la producción agrícola, pecuaria y el turismo, donde sobresale por su belleza natural y su accesibilidad la cascada El Vino.

La escuela granja Hato Arriba, fundada en 1960, contaba a la fecha de realización de la investigación con una población de 409 habitantes, de los cuales 348 estudiantes pertenecían a los subsistemas educativos inicial, primaria y secundaria. Asimismo, se atendían a 240 estudiantes semi-internos y 108 estudiantes internos provenientes de las comunidades de otras parroquias, así como de otros municipios. Además, del personal directivo conformado por 3 profesores y el personal docente con 36 profesores de aula; le sigue el personal administrativo comprendido por 4 personas y por último, el personal obrero con 21 trabajadores. 
Por otra parte, la actividad socioeconómica del sector se fundamenta en la producción agropecuaria tanto vegetal en los rubros hortícolas, como animal, en la elaboración de subproductos lácteos para la venta tales como: la cuajada, el queso, suero, leche, dulces, y las carnes. En tal sentido, la escuela como centro del quehacer comunitario toma el rumbo del perfil agropecuario y se encuentra en una transición de transformación a escuela técnica agropecuaria debido a las características de la zona antes mencionadas. Asimismo, la comunidad en general participa en la conformación de cooperativas agrícolas, creación de tres consejos comunales y la escuela como entidad, tiene registrada la Asociación Civil de Padres y Representantes que le permite llevar los registros de las compras y ventas legales de cualquier transacción comercial.

En el mismo orden de ideas, la escuela cuenta con los servicios públicos como agua, energía eléctrica, teléfono prepago y/o celulares del personal, internet por modem prepago, transporte por alquiler de la comunidad educativa en camiones 350; y carece de los servicios básicos de transporte público, tratamiento de aguas servidas y salud especializada. No obstante, en cuanto a la atención sanitaria, el médico del plantel durante el año escolar realiza consultas a la comunidad una vez por semana.

El interés de los autores por el área de estudio surgió de la observación de las características físico naturales boscosa-montanas de clima fresco donde se ubica la comunidad educativa, asociadas a las conductas observadas en los estudiantes en cuanto a la conservación del entorno.

\section{El Procedimiento Seguido}

La presente investigación fue elaborada bajo el enfoque cualitativo apoyada en el método fenomenológico (Martínez, 2004b) ya que se adecúa al objeto de estudio por permitir entender las realidades de los actores desde sus percepciones sobre la dimensión ambiental en el comportamiento cotidiano del estudiante de la tercera etapa de educación básica, de la Escuela Granja Hato Arriba ubicada en el caserío de nombre similar en la parroquia Morán del Municipio Morán, Estado Lara. Se concibe como un estudio del quehacer frecuente de los individuos o grupos donde se toma en cuenta el respeto por lo 
que la gente expresa, piensa o siente, así como sus estándares culturales y el significado de sus relaciones interpersonales y con el hábitat.

Asimismo, se utilizaron como técnicas para la recopilación de información a la observación participativa, la toma de fotografías y las entrevistas a profundidad, lo cual contribuyó a la construcción del pertinente marco interpretativo desde la percepción de los actores educativos de la comunidad educativa de la escuela granja Hato Arriba.

La selección de los informantes clave de la comunidad educativa antes mencionada, se realizó mediante el muestreo intencional de acuerdo a los siguientes criterios: a) género, b) edad, c) niveles de conocimiento sobre educación ambiental y d) nivel de conocimiento sobre conservación ambiental. De esta manera se entrevistaron 19 miembros representativos de la comunidad educativa, con quienes se logró la saturación de la información de acuerdo a lo señalado por Glasser y Strauss (1967) y Bertaux (1999).

Para el desarrollo de este estudio los autores recurrieron al procedimiento sugerido por Martínez (op. cit, b) para la investigación fenomenológica, estructurado en las siguientes etapas: a) etapa previa o de clarificación de los presupuestos, consistente en una fase de acercamiento, revisión e inserción en la problemática investigativa, b) etapa descriptiva, tendiente a la elaboración de una descripción lo más completa posible del fenómeno en estudio, desde la perspectiva de los informantes, c) etapa estructural, en la cual se realiza una aproximación interpretativa de las descripciones comprendidas en los protocolos, así como la construcción de las estructuras particulares y general del fenómeno estudiado. Una vez terminadas las tres etapas, se ejecutó la discusión de los hallazgos, en la cual se procedió a la reflexión personal y grupal sobre los hallazgos pertinentes a la investigación. En el Cuadro 1 se muestran los detalles correspondientes.

En el mismo orden de ideas, es importante señalar nuestro énfasis como investigadores en el desarrollo del proceso de investigación de acuerdo a las orientaciones y recomendaciones sugeridas por Martínez (op. cit. b): el nivel de precisión terminológica, el rigor metodológico en cuanto a la adecuación del método al objeto, la sistematización para el desarrollo del proceso y la actitud crítica que acompaña al citado proceso investigativo6. 


\section{LOS HALLAZGOS}

En este punto se presentan los hallazgos de la investigación, producto de la aplicación del enfoque metodológico mencionado previamente. Es importante señalar el surgimiento de las siguientes categorías interpretativas, a partir de revisión de los protocolos de las entrevistas: comportamiento cotidiano, conservación ambiental, conflicto y la educación ambiental. Así, es posible mencionar al comportamiento cotidiano, como la categoría principal más resaltante, tal cual como lo indican los informantes:

"Esteee, bueno en veces más o menos me comporto más o menos, cuando yo compro algo en la cantina los papeles los echo en la papelera. Y cuando ando con los muchachos lo echamos pa' la paja” (Informante I2).

Asimismo, el informante I1 manifestó lo siguiente:

...si estoy cerca de la casa lo echo en...en una papelera...o si 'toy por fuera lo echo así pa’l monte... porque a veces me provoca y lo tiro...lo tiro en la carretera, o si es una botella de vidrio, lo tiro pa'l monte...Porque alguna veces uno va pasando y se le olvida y se está comiendo la chuchería y bota el papel en la carretera.

Durante su estadía como docente en la escuela granja Hato Arriba, uno de los autores ha podido observar a los estudiantes lanzar los desechos de las golosinas o chucherías y desperdicios sólidos fuera de las papeleras, por los alrededores de las áreas administrativas e incluso del mismo comedor, así como también, en las cercanías de la cantina y las aulas de clases; esto ha generado un comportamiento cotidiano que repercute en los otros compañeros de clases quienes, en su compartir, casi siempre terminan imitando y actuando como sus semejantes. Al respecto, otro de los informantes señaló:

Bueno, mucha falta de conciencia, ellos no ponen conciencia donde lo van a tirar a pesar de que ponen papeleras en todas partes, será que no las ven o se hacen los que no las ven y eso es falta de conciencia... Cuando se lo comen allá mismo en el patio que está al frente de la cantina lo adornan con papeles de servilletas, de caramelos, de todas las golosinas que compran y cuando se lo van a llevar a otras áreas lo tiran al suelo o debajo del pupitre, encima del pupitre, ahí lo dejan y si van vía a los salones lo tiran al suelo (Informante I19). 


\section{Cuadro 1}

\section{Procedimiento de la Investigación}

\begin{tabular}{|c|c|}
\hline Etapas & Pasos \\
\hline $\begin{array}{l}\text { Etapa previa o de clarificación } \\
\text { de los presupuestos }\end{array}$ & $\begin{array}{l}\text {-Revisión de las creencias, actitudes, presupuestos, intereses y } \\
\text { conjeturas, para puntualizar como influyen en la investigación. Durante } \\
\text { esta etapa se realizaron visitas al área de estudio así como entrevistas } \\
\text { iniciales con cuatro informantes claves y se elaboraron los protocolos de } \\
\text { investigación respectivos. }\end{array}$ \\
\hline Etapa Descriptiva & $\begin{array}{l}\text {-Elección de la técnica o procedimientos a través de la observación directa } \\
\text { dentro de la comunidad educativa, tomando notas y recogiendo datos. } \\
\text { - Entrevista a profundidad que Martínez (2002 a) define como "una } \\
\text { forma de diálogo coloquial flexible y dinámica, abierta, donde el } \\
\text { entrevistado tiene la oportunidad de hablar libremente” (p. 142). Es } \\
\text { importante señalar la realización de grabaciones de las conversaciones, } \\
\text { toma de fotografías y preparación de un guión de entrevistas para la } \\
\text { construcción posterior de las estructuras particulares y general del } \\
\text { fenómeno estudiado. }\end{array}$ \\
\hline Etapa Estructural & $\begin{array}{l}\text {-Elaboración de la descripción protocolar: Desarrollo de las observaciones } \\
\text { reflejando la realidad tal cual como se presenta; transcripción de las } \\
\text { entrevistas respetando las expresiones y opiniones de los informantes } \\
\text { clave. Esta etapa se desarrolló de acuerdo con los siete pasos } \\
\text { recomendados por Martínez (op. cit. b): } \\
\text { 1. La descripción de los protocolos para lograr la idea general del } \\
\text { contenido. } \\
\text { 2. Delimitación de las unidades temáticas naturales. } \\
\text { 3. Determinación del tema central que domina cada unidad } \\
\text { temática. } \\
\text { 4. Expresión del tema central en lenguaje científico. } \\
\text { 5. Integración de todos los temas centrales en una estructura } \\
\text { particular descriptiva. } \\
\text { 6. Integración de todas las estructuras particulares en una estructura } \\
\text { general. } \\
\text { 7. Entrevista final con los sujetos estudiados, para darles a conocer } \\
\text { los hallazgos de la investigación. }\end{array}$ \\
\hline $\begin{array}{l}\text { Discusión de resultados o } \\
\text { hallazgos }\end{array}$ & $\begin{array}{l}\text {-Cumplidas las tres etapas, se procedió a la discusión de los hallazgos, así } \\
\text { como su contrastación con los hallazgos y reflexiones de otros } \\
\text { investigadores, mediante comparaciones y contraposiciones, para entender } \\
\text { las posibles diferencias y así llegar a una integración mayor. }\end{array}$ \\
\hline
\end{tabular}

En relación a lo antes expuesto, existen otras opiniones en las cuales no se involucra a todos alumnos en este comportamiento o conducta en cuanto a la deposición de los desechos sólidos. Al respecto, el informante I15 señala que “...son algunos que tienen ese comportamiento usted sabe que no todos se comportan como debe ser. A veces lo hacen como a propósito, otros lo hacen sin conciencia...” 
Asimismo, otro de los entrevistados se refirió al comportamiento cotidiano de los estudiantes de la siguiente manera: "Bueno, se ha notado que en la mayoría de los estudiantes la primera reacción al comprar es tirar el desperdicio al piso, cualquier desecho que tienen lo tiran al piso” (Informante I17).

De esta manera, conductas como las señaladas por los actores se observan día a día en el plantel, tal es el caso del siguiente sujeto quien manifestó lo siguiente: "Lo echo en la papelera...pero yo igualito lo tiro a veces en el piso... porque me da pereza ir ahí donde está la papelera...y algunas veces lo tiro así en el monte.” (Informante I3).

Por otra parte, es relevante hacer referencia a los diversos conflictos que se generan entre las y los estudiantes con los obreros, principalmente, en torno a la deposición de los desechos sólidos en las distintas áreas del plantel, sin obviar que día a día el docente se encuentra en constante atención para corregir estas conductas de estos actores educativos. Al respecto, uno de los informantes plantea:

...los alumnos así haiga las papeleras ellos se comen las empanadas y tiran los papeles fuera de la papelera verdad, y entonce le dice no, no lo hagan, métanlo en la papelera eso se ve feo ahí...Pero uno les dice a ellos y ellos se portan algunas veces malcriados con uno, le dicen...que te importa a ti eso. (Informante I14).

En el mismo orden de ideas, el personal obrero manifestó el descontento por la actitud inadecuada del estudiantado en relación a su ambiente de clase. Al respecto, uno de los informantes manifestó: “...bueno, unos aceptan la corrección y otros no, me miran y dicen: ¡eso lo recoge el obrero!, o dicen: ¡otro papelito más eso no quiere decir nada!...” (Informante I16), en este sentido, el mismo informante expresa lo siguiente:

...Me pasó un caso con una niña...llevaba un envase...lo arroja hacia a fuera hacia el monte, le digo que eso no se debe hacer... ella dice: ique por ese envase...que ella arrojó no se va a acabar el mundo, que muchas personas también lo hacen!...

En este sentido, expresiones como estas son comunes en la mayoría de las y los estudiantes de la institución por lo que genera enfrentamientos entre los actores educativos y las aseadoras básicamente; así lo manifestó la siguiente entrevistada: 
Bueno, yo le llamo la atención al niño, le hablo de que eso es contaminar el ambiente y que para eso existen las papeleras... hay unos que reciben la corrección bien y hay otros que de pronto se alteran, no les gustan que uno les llame la atención (Informante I17).

En concordancia con lo expuesto anteriormente, el informante I14 expresó lo siguiente:

...Pasa lo siguiente aquí en la escuela, se comen una arepa cuando se van a ir y eso hacen una reguera, cuando le dan el jugo eso lo dejan un chiquero, ique trabajen las mujeres del aseo!, es lo que dicen.

Por otra parte, es importante señalar que en la institución constantemente se habla a la población estudiantil de los tres subsistemas sobre la educación ambiental y la conservación ambiental, sin embargo, existe un grupo significativo de esta población que aún no asimila dicha información como se ha señalado en el desarrollo de la investigación. Ante lo expuesto, el informante I4 expresó la importancia que tiene el conservar el ambiente explicándolo de la manera siguiente:

...Estamos limpiando toda la escuela, agarrando todas las partes de sucio, estamos 'onde hay matas así, 'tamos sembrando, sembrando para tener este ambiente, tener la escuela bien boni... bien, bien bonita pues y tener así, construir el ambiente y por aquí el director, profesores, cocineras, obreros, le dicen a uno epa esto va aquí esto va allá, se acuerdan del club ambiental...este uno mismo... este usted ayuda y después hace, hace lo contrario empieza a botar la basura donde mismo, donde mejor le parezca las cosas no deberían ser así, debemos tomar conciencia sobre eso.

Asimismo, uno de los entrevistados del personal docente resaltó el trabajo que se ha venido realizando dentro del plantel al afirmar: “...Si, se han realizado carteleras alusivas, el año pasado se hizo una cartelera sobre la destrucción de la capa de ozono, lo del calentamiento global y otras, si se le ha hecho propaganda a la conservación del ambiente” (Informante I18).

De esta manera, según lo observado, la institución busca concienciar al estudiantado así como a todo el personal y la comunidad educativa en general para el logro de un comportamiento significativo ambientalista que permita conservar el entorno donde se 
desarrollan los procesos educativos con el apoyo de la dimensión ambiental. Además, es relevante mencionar la alusión constante a la conservación ambiental dentro del plantel, en vista de la realización de charlas, campañas como la Semana de la Conservación Ambiental, tardes ecológicas; no obstante, el estudiantado en general siempre muestra una conducta ambientalista con bajo compromiso, en cuanto a la conservación ambiental del entorno. Sobre este particular, se manifestó la siguiente expresión por parte de uno de los sujetos entrevistados:

$\mathrm{Si}$, desde siempre a pesar de que ellos tienen materias que se relacionan con la contaminación del ambiente incluso hasta la profesora de inglés, matemática que no tienen nada que ver, que no tienen que dar una clase de ambiente, siempre les están dando charlas sobre proteger y conservar el ambiente y que una de las principales contaminantes del ambiente es la basura (Informante I19).

Ante lo expuesto anteriormente, se pudo observar en la mayoría de las y los estudiantes una débil conciencia sobre lo concerniente al tema ambiental, dadas las manifestaciones de una conducta inadecuada al momento de consumir los alimentos y golosinas de manera muy frecuente, dentro y fuera de los ambientes de clases.

En el mismo orden de ideas, al preguntarle a uno de los informantes si conocía sobre educación ambiental manifestó lo siguiente: “...Este, no debemos tirar nada por donde, por ahí y debemos cuidar el ambiente, en la escuela esteee...mm... que debemos cuidar y no dañar las cosas y y, mm... este no botar, no botar basura” (Informante I5), en concordancia con lo antes expuesto, otro informante expresó: “Que por lo menos uno no debe andar botando los papeles así, que uno debe decirle que, que no lo bote, que eso después lo va afectar mucho el ambiente” (Informante I6), asimismo, otro de los entrevistados manifestó conocer al respecto de la educación ambiental lo siguiente:

Se yo sobre educación ambiental queee...no no tirar los papeles así al al piso, no quemar los plásticos porque esos contaminan el aire, no cortar los árboles porque secan el agua; el profesor Jorge nos inculca de eso porque nos dice en educación para el trabajo, si uno bota un papelito recojan ese papelito porque eso porque eso eso daña la Tierra (Informante I8).

En este sentido, se puede evidenciar los pocos conocimientos que manejan el estudiantado al referirse a la educación ambiental como normativas a seguir para conservar 
el entorno donde realizan sus actividades académicas. Al respecto, el informante I9 manifestó:

Bueno, los profesores dicen mmm...sobre todo en biología, este debemos agarrar las cosas que van en la basura para así no contaminar el ambiente, porque si contaminamos el ambiente, igual nos contaminamos nosotros, porque el aire que nosotros respiramos está contaminado.

De acuerdo a lo expresado anteriormente, se observó que la mayoría de los docentes y obreros están tratando de concienciar a las y los estudiantes de la escuela granja Hato Arriba, debido al bajo nivel de conocimientos y de conciencia de estos estudiantes sobre el significado de la educación ambiental. Al respecto, el informante I7 expresó “Que no se debe contaminar el ambiente...Botar la basura y el ambiente se puede contaminar y se puede acabar el planeta Tierra...”.

Al respecto, el informante I10 expresó: “Que todos mm...tengamos quee echar los papeles donde van y no echarlos en el suelo y mm...que cuando hay papeles que lo recojan...”, otro de los entrevistados indicó: “...que por lo menos una planta que uno le tire basura la planta se daña... porque la naturaleza es un ser como nosotros, la naturaleza vive” (Informante I11). Asimismo, el informante I12 manifestó haber oído lo siguiente sobre la educación ambiental:

...Bueno es donde se le inculcan valores a los estudiantes, donde se motivan, donde se motivan a que sean bueno ciudadanos, donde les llame la atención, cuidar la naturaleza; es donde se le inculcan una serie de valores para ponerlo en práctica con la naturaleza.

En el mismo orden de ideas, durante las entrevistas realizadas la mayoría del estudiantado concibe la educación ambiental de la siguiente manera: "No dañar las plantas... la educación ambiental entiendo que...gueno, uno no debe tira la basura en donde le provoque sino en la papelera” (Informante I13).

Por otra parte, en el Cuadro 2 se muestra la estructura general del fenómeno estudiado el cual surgió de las estructuras particulares construidas a partir de las revisiones de los protocolos de las entrevistas y del surgimiento de las categorías interpretativas mediante la comprensión de las estructuras particulares surgidas para cada informante: comportamiento cotidiano, conservación ambiental, conflicto y educación ambiental. 
Sobre este particular, surgió el octaedro de Von Eckartsberg (Martínez, op. cit.b), en relación a la dimensión ambiental y comportamiento cotidiano, en la que se plantean ocho interrogantes fundamentales como son: ¿por qué?, ¿a qué nivel?, ¿cómo?, ¿a quién?, ¿cuándo?, ¿dónde?, ¿qué? y por último, ¿quiénes?. Dichas interrogantes facilitaron la ampliación de la descripción de la estructura general, ya que a través de ellas se logró visualizar las actividades de los coinvestigadores, así como también complementó y puntualizó los detalles de la estructura y por ello fortalecí el fenómeno investigado; por otra parte, ayudó a integrar todas las acciones de los actores educativos de esta comunidad.

Por consiguiente, se asociaron las categorías de las estructuras particulares y de la general, las cuales emergieron durante el desarrollo de la investigación, donde se satura la información, debido a la coincidencia en las respuestas de los informantes, emanadas de las de las entrevistas realizadas, en relación al comportamiento cotidiano que presentan las y los estudiantes de la comunidad educativa y sus respectivas causas; las consecuencias de tales comportamientos, y la percepción sobre la necesidad de la existencia de un mayor compromiso e identidad por parte de los docentes y los otros actores educativos en la aplicación de la dimensión ambiental, tal como se presenta en el Gráfico 1. Adicionalmente, se muestra en el Gráfico 2 la representación esquemática de la estructura general del fenómeno investigado.

Los hallazgos y su contrastación con los referentes teóricos fueron presentados a los integrantes de la comunidad educativa, quienes mostraron su acuerdo con los contenidos mostrados.

La infraestructura física de la institución, en su mayoría, data de los años 50, y fue construida inicialmente para servir como hacienda privada de la familia Tamayo Suárez. Posteriormente, estos espacios físicos fueron acondicionados para las actividades educativas para los subsistemas de educación inicial, primaria y secundaria, este último desde el año 2007 cuando se dio inicio al cuarto año técnico, con el propósito de capacitar al estudiantado ciencias agrícolas hasta el sexto (6to) año técnico, para su egreso como técnicos medios profesionales. 


\section{Cuadro 2}

\section{Estructura General del Fenómeno Estudiado}

\begin{tabular}{|c|c|c|c|c|}
\hline & \multicolumn{4}{|c|}{$\begin{array}{l}\text { Aspecto: La Dimensión Ambiental en el Comportamiento Cotidiano del Estudiante de la Tercera Etapa de } \\
\text { Educación Básica. }\end{array}$} \\
\hline & Comportamiento cotidiano & Conservación ambiental & Conflictos & Educación ambiental \\
\hline$\frac{1}{d}$ & $\begin{array}{l}\text { No existe congruencia en el } \\
\text { comportamiento cotidiano por } \\
\text { parte del estudiante, al disponer } \\
\text { de los desechos sólidos en sitios } \\
\text { inapropiados para tal fin, dentro } \\
\text { y fuera del plantel. Algunos de } \\
\text { las y los estudiantes por } \\
\text { inconsciencia o por flojera } \\
\text { depositan los residuos en la } \\
\text { vegetación, carreteras, pisos y } \\
\text { aulas sin tener respeto a su } \\
\text { entorno. Asimismo, se evidencia } \\
\text { una conducta impropia con } \\
\text { mayor ímpetu en los grupos que } \\
\text { en relación al comportamiento } \\
\text { individual. Por otra parte, existe } \\
\text { una falta de conciencia ambiental } \\
\text { que debe partir desde el hogar ya } \\
\text { que si se comportan así es una } \\
\text { cuestión de cultura. }\end{array}$ & $\begin{array}{l}\text { Para conservar el ambiente } \\
\text { se debe expresar en } \\
\text { acciones ambientalistas con } \\
\text { conciencia que evite el } \\
\text { deterioro del entorno. Tales } \\
\text { acciones pueden ser evitar } \\
\text { la disposición de los } \\
\text { desechos sólidos en sitios } \\
\text { inadecuados, recolección de } \\
\text { los residuos para conservar } \\
\text { el medio. Por una parte, se } \\
\text { evidencia la falta de valores } \\
\text { desde el hogar, asimismo en } \\
\text { la escuela ya que no existe } \\
\text { el compromiso por parte de } \\
\text { los docentes para enfocarse } \\
\text { en formar en valores éticos } \\
\text { ambientalistas y así } \\
\text { conservar el entorno donde } \\
\text { interactúan. }\end{array}$ & $\begin{array}{l}\text { Los desechos sólidos } \\
\text { son dispuestos por } \\
\text { algunos de las y los } \\
\text { estudiantes donde les } \\
\text { provoca o porque se les } \\
\text { pasa por alto y en } \\
\text { reiteradas ocasiones, } \\
\text { siendo sitios } \\
\text { inadecuados para tal fin. } \\
\text { Esta acción contamina el } \\
\text { entorno lo que ocasiona } \\
\text { un descontento en el } \\
\text { personal obrero de aseo } \\
\text { cuando le llaman a la } \\
\text { reflexión para que no } \\
\text { tiren los desechos } \\
\text { sólidos en el piso, en } \\
\text { cualquier sitio aun } \\
\text { sabiendo la existencia de } \\
\text { las papeleras dentro del } \\
\text { plantel. }\end{array}$ & $\begin{array}{l}\text { En la escuela los docentes } \\
\text { les inculcan a las y los } \\
\text { estudiantes sobre la } \\
\text { educación ambiental. La } \\
\text { preocupación por } \\
\text { alertarlos como debe } \\
\text { hacerse con la disposición } \\
\text { de los desechos sólidos en } \\
\text { los sitios adecuados para } \\
\text { tal fin, así como los } \\
\text { distintos contenidos en } \\
\text { materia de conservación } \\
\text { ambiental. Asimismo, le } \\
\text { recuerdan que un residuo } \\
\text { sólido en el suelo } \\
\text { cultivable lo contamina y } \\
\text { por ello no se deben } \\
\text { depositar allí. Al } \\
\text { respecto, la educación } \\
\text { ambiental es donde se } \\
\text { motiva y se inculcan una } \\
\text { serie de valores para ser } \\
\text { buenos ciudadanos y } \\
\text { ponerlos en práctica con } \\
\text { la naturaleza. }\end{array}$ \\
\hline
\end{tabular}

Al respecto, de acuerdo a los registros de la institución se cuenta con una matrícula total de 358 estudiantes provenientes de diversas parroquias del estado Lara, desde la ciudad de Barquisimeto, El Tocuyo, los Humocaros Bajo y Alto, y de los caseríos aledaños al plantel como La Laguna, La Yerba Buena, La Cebada, El Cujizal, San Pedro entre otros. Por tal motivo, el plantel maneja la modalidad de internado, atendiendo una matrícula de internos y semi-internos. 


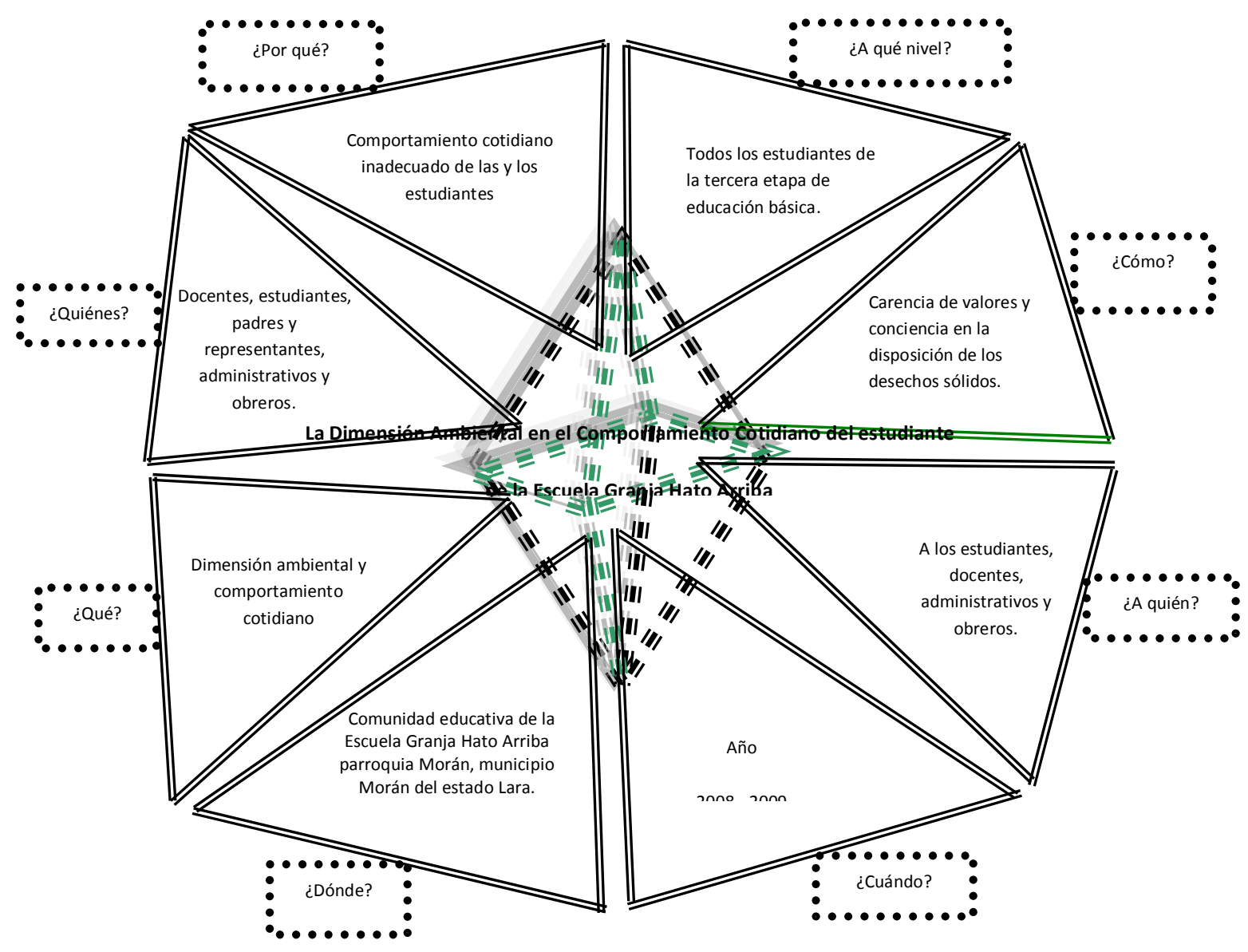

Gráfico 1. Octaedro: La Dimensión Ambiental en el Comportamiento Cotidiano del Estudiante de la Escuela Granja Hato Arriba.

Es importante mencionar, que veintisiete de los docentes pernotan toda la semana en la escuela, dada la existencia de residencias tanto femenina como masculina. Sin embargo, a pesar de convivir gran parte del tiempo con las y los estudiantes y desarrollar actividades conmemorativas como el Día Mundial del Agua, la Semana de la Conservación, Día al Árbol, Día Mundial del Ambiente, entre otras, el nivel de consciencia es débil por lo que el comportamiento cotidiano de estos adolescentes es inadecuado. En tal sentido, se evidencia la falta de interés de los docentes en cuanto al compromiso de vivenciar, experimentar y transmitir a los educandos una conducta apropiada en materia de conservación ambiental. 


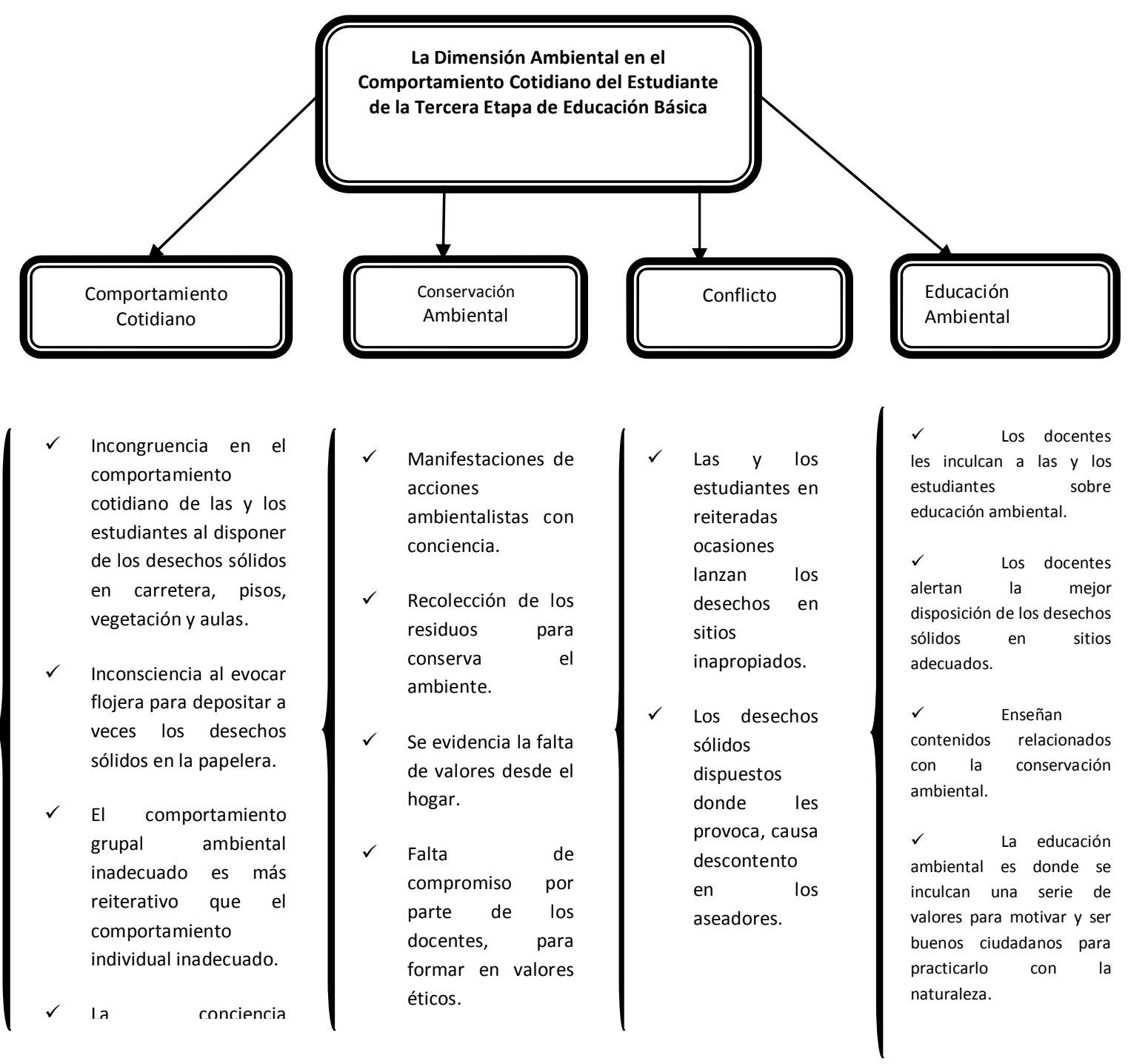

Gráfico 2. Presentación Esquemática de la Estructura General del Fenómeno Estudiado

En relación a la problemática observada se pude constatar la actuación de las y los estudiantes a la hora de comprar en la cantina y en las horas de comedor, en cuanto a la manera de disposición de los desechos sólidos en el suelo y pasillos de las áreas

administrativas, dentro de las aulas de clases y en las áreas verdes. De esta manera se señala la concordancia de los comportamientos de los estudiantes con lo expresado por Uribe, Velasco y Escobar (1991), en cuanto a la poca una conciencia del efecto que causamos sobre nuestro entorno, tanto cuando actuamos a nivel personal como cuando emprendemos una acción colectiva y por ello aún nos mantenemos limitados para evaluar de manera 
adecuada el impacto ambiental que estamos generando y que, como todos sabemos tiene varios posibles caminos de deterioro sin retorno.

De igual manera, surgió la importancia de la consideración de la psicología ambiental como disciplina de apoyo para los docentes en el proceso de transformación de los educandos hacia una conducta ambientalista y a su vez, con el apoyo de la educación ambiental, para que ayuden con hechos vivenciales que puedan conducir a los educandos a ser concientizados y así dejen de contaminar el ambiente al disponer de los desechos sólidos en sitios inadecuados (Zanzíbar en Baldi y García, 2005; Covas, 2004).

\section{REFLEXIONES FINALES}

En el área de estudio existe indiferencia por parte del estudiantado en la disposición de los desechos sólidos en los distintos lugares ambientes donde se encuentren en la institución. Asimismo, es manifiesta la carencia de compromiso por parte de los docentes en materia de educación ambiental, quienes en general sólo buscan cumplir académicamente con los programas establecidos y no dan el valor agregado para concienciar al estudiante, por lo que se dificulta la transformación de las conductas en el estudiantado de manera significativa en lo referente a la conservación ambiental.

Es indudable la necesidad de la existencia de un mayor compromiso e identidad por parte de los docentes, padres y representantes además de los otros actores educativos en la difusión de la dimensión ambiental entre los estudiantes, visto el débil interés en el mejoramiento del comportamiento cotidiano de estos actores educativos y cual a su vez, al fortalecerse pudiera contribuir a la mitigación de los diferentes problemas ambientales presentes en la comunidad educativa estudiada. Aunado a lo anterior, se observó la falta de valores desde el hogar, aspecto de mucha relevancia para lograr forjar el carácter de un individuo desde temprana edad.

De esta manera, la construcción de un marco interpretativo desde la percepción de los actores de la comunidad educativa de la escuela granja Hato Arriba, impulsó a indagar sobre la percepción de los actores educativos desde la interacción entre si y con el ambiente, el punto de vista compartido de una problemática en la disposición de los desechos sólidos en sitios inadecuados por todo el plantel educativo, motivado por la 
dimensión ambiental en el comportamiento cotidiano de las y los estudiantes, a través de la interpretación de la percepción de dichos actores en relación al comportamiento cotidiano diferenciado en los distintos ámbitos de la comunidad educativa, construyendo así las estructuras particulares y general describiendo en éstas, la fisonomía individual y luego la estructura fisonómica que caracteriza al grupo estudiado, todo esto originado desde las unidades temáticas donde se describen la naturaleza de los protocolos.

Como resultado de la observación participante y de la comprensión de la información suministrada por los informantes clave, la cual sirvió para indagar a profundidad sobre la dimensión ambiental, para luego interpretar y construir la esencia del fenómeno estudiado, se puede manifestar lo siguiente:

1. Al indagar sobre la percepción de los actores educativos sobre sus experiencias y vivencias en cuanto a la dimensión ambiental del comportamiento cotidiano del educando en la comunidad educativo antes mencionada, se develó la existencia de una incongruencia con el deber ser, vista la tendencia de la mayoría de las y los estudiantes para disponer los desechos sólidos en sitios inapropiados para tal fin, aún contando con las papeleras, situación que conlleva a la contaminación del entorno educativo.

2. La interpretación de la percepción desde los mismos actores educativos sobre la dimensión ambiental en el comportamiento cotidiano de las y los estudiantes observado en el plantel, condujo a señalar la deficiencia ambiental existente en los docentes para comprometerse con la educación ambiental como herramienta pedagógica para lograr la conservación ambiental, en vista de que solamente cumplen con un contenido programático y académico predefinido por las autoridades educativas nacionales.

3. Asimismo la interpretación de las percepciones de las y los informantes permitió visualizar la deficiencia ambiental presente en ellos por no tener conciencia a la hora de su alimentación, durante las tres ingestas suministradas por el plantel, además de las respectivas meriendas suministradas, para la disposición de los desechos sólidos en los sitios adecuados en los diferentes ámbitos de la comunidad educativa, lo que genera conflictos con el personal obrero.

4. Al construir las estructuras particulares y general se pudo identificar las fisonomías individual y grupal del comportamiento cotidiano de las y los estudiantes presentándose una mayor congruencia en los comportamientos grupales para accionar 
inadecuadamente al consumir alimentos y golosinas, diferenciadas en los distintos ámbitos de la comunidad educativa.

En relación a los conflictos señalados, se está desarrollando la conformación de la brigada ambientalista de la escuela granja Hato Arriba, con el propósito de concienciar primero a los actores educativos y seguidamente a los habitantes de la comunidad educativa y en general. De esta manera, se pretende fortalecer los lazos de participación colectiva donde interactúen las y los estudiantes, personal docente, obreros, administrativos, directivos, padres y representantes que ayuden a mejorar las relaciones interpersonales en pro de conservar su hábitat y elevar la conciencia ambientalista deseada.

Producto de las reflexiones antecedentes, es posible plantear las siguientes recomendaciones:

1. Es necesario el compromiso y el sentido de pertenencia por parte de los docentes, personal obrero, administrativo, padres y representantes, personal directivo que apoyen el proceso de transformación del comportamiento cotidiano de las y los estudiantes de manera positiva para la conservación de todos los espacios donde interactúan.

2. Es recomendable que las autoridades educativas como el Ministerio del Poder Popular para la Educación, la Zona Educativa y la Dirección Municipal de Educación, brinden el acompañamiento del proceso de actuación colectiva en la conservación ambiental del sector.

3. Por lo antes señalado, se sugiere la conformación de una contraloría social ambientalista que mantenga un acompañamiento de la inserción de la educación ambiental en cada subsistema y asegure la aplicación de dicha dimensión ambiental.

4. Se recomienda a los docentes del plantel, la elaboración académica de proyectos de aula concernientes al perfil ambiental, donde interactúen la escuela y la comunidad para preservar y conservar los espacios naturales.

5. Se propone la elaboración de actividades extra cátedras para concienciar a la comunidad educativa en general que les permitan realizar proyectos ambientalistas para el mejoramiento de la calidad de vida.

6.- Finalmente se recomienda darle continuidad a este trabajo de investigación para darle el seguimiento y obtener mejores resultados en relación a la dimensión ambiental 
en el comportamiento cotidiano del estudiante de la tercera etapa de educación básica de dicha comunidad educativa.

\section{REFERENCIAS}

Alea, A. (2005). Introducción a la psicología ambiental. [Documento en línea]. Disponible: www.monografias.com/trabajos26/psicologiaambiental/psicologia-ambiental.shtml [Consulta: 2008, Septiembre 12].

Baldi, G. y García, E. (2005). Calidad de vida y medio ambiente: Psicología ambiental. En Redalyc 030, [Revista en línea].Disponible: redalyc.uaemex.mx/redalyc/pdf/373/37303003.pdf- [Consulta: 2008, Septiembre 20].

Bertaux, D. (1999). El enfoque biográfico: Su validez metodológica, sus potencialidades. [Artículo en línea]. Traducido por la Universidad de Costa Rica en 1999. Disponible: http://www.scribd.com/doc/6901568/Bertaux-D-1999 [Consulta: 2009, Julio 2].

Covas, O. (2004). Educación ambiental a partir de tres enfoques: comunitario, sistémico e interdisciplinario. En Revista Iberoamericana de Educación, 34 [Revista en línea]. Disponible: www.rieoei.org/deloslectores/794Covas.PDF -. [Consulta: 2008, Agosto 20].

Escalona, J., y Pérez, M. (2006). La educación ambiental en la Universidad de Los Andes: Un estudio desde la perspectiva de los estudiantes de educación. [Documento en línea].

Disponible:http://www.scielo.org.ve/scielo.php?pid=S131649102006000300011\&scrip t=sci_arttext. [Consulta: 2008, Septiembre 29].

Ferrer, E. (2001). Conservación ambiental: Alternativa para el futuro. Serie: Ecología y conservación ambiental. Barquisimeto, Lara: Ecosmos.

Fernández, R., Rodríguez, L y Carrasquer, J. (2006). El conocimiento de las actitudes ambientales: una buena base para mejorar las conductas hacia el medio ambiente. III jornadas de educación ambiental de la comunidad Autónoma de Aragón, España. [Documento en línea].Disponible: portal.aragon.es/portal/page/portal/MEDIOAMBIENTE/EDUAMB/SENSIBILIZACI ON/.../ACTITUDESAMBIENTALES.PD... -. [Consulta: 2008, Octubre 21].

Flen-Bers, M. (2001). Actitud del docente preescolar frente a la educación ambiental. Trabajo especial de grado no publicado, La Universidad del Zulia, Maracaibo. [Documento en línea]. Disponible:www.monografias.com/trabajos14/docentepreesc/docentepreesc.shtml _ 57k. [Consulta: 2008, Noviembre 11]. 
García, G. (2008). Consideraciones teóricas en torno al tema de los grupos. [Documento en línea]. Disponible: www.monografias.com/trabajos15/psico-med-ambiente/psicomed-ambiente.shtml - 66k -. [Consulta: 2008, Agosto 29].

Glaser, B. y Strauss, A. (1967). The discovery of grounded theory: Strategies for qualitative research. New York: Aldine de Gruyten.

Gutiérrez, D. (2007). Nivel de información sobre conservación ambiental y la actitud conservacionista del estudiante de la II Etapa de Educación Básica de la Parroquia Buria del Municipio Simón Planas del Estado Lara. Trabajo de grado de maestría no publicado, Universidad Yacambú, Barquisimeto.

Ley Orgánica del Ambiente (2006). Gaceta Oficial de la República de Venezuela, 5.833 (Extraordinario), Diciembre 22, 2006.

Martínez, M. (2002a). La nueva ciencia. México: Trillas.

Martínez, M. (2004b). Comportamiento humano. (2ªed.) México: Trillas.

Mora, C. (2005). Gestalt y comportamiento organizacional. [Documento en línea].Disponible: $\quad$ http://www.gestiopolis.com/canales5/rrhh/gestatproganiza.htm. [Consulta: 2008, Agosto 29].

Moreno, M., Corraliza J. y Ruiz J. (2005).Escala de actitudes ambientales hacia problemas específicos. En Psicothema 03, [Revista en línea]. Disponible: www.psicothema.com/psicothema.asp?id=3136 - 56k -. [Consulta: 2008, Noviembre $11]$.

Pato, C. y Tamayo, A. (2006). Valores, creencias ambientales y comportamiento ecológico de activismo. [Documento en línea]. Disponible: webpages.ull.es/users/mach/PDFS/Vol7_1/Vol7_1_d.pdf -. [Consulta: 2008, Octubre 21].

Tréllez, E. (2000). Manual guía para comunidades educación ambiental y conservación de la biodiversidad en el desarrollo comunitario. [Documento en línea]. www.comunidad.info/presta,php?ele=314 [Consulta: 2008, Agosto 23].

Uribe, C., Velasco, J. y Escobar, J. (1991). Evaluación de impacto ambiental como un componente de la evaluación económica. . [Documento en línea]. Disponible: $\quad$ http://www.cepis.ops-oms.org/eswww/fulltext/repind51/cee/cee.html. [Consulta: 2008, Diciembre 18].

VITALIS. (2007a). Diccionario digital en internet. [Documento en línea]. Disponible: http://www.vitalis.net/Glosariob.htm - 110k. [Consulta: 2007, Octubre 12]. 
Zaldívar, D. (2003). Psicología y medio ambiente. En Infomed Ambiente Salud Vida, 17. [Revista en línea]. Disponible: www.sld.cu/saludvida/ambiente/temas.php?idv=5828 [Consulta: 2008, Agosto 10] 